\title{
Sigma-Delta ADC Based Distributed Detection in Wireless Sensor Networks
}

\author{
Dimeng Wang, Shuangqing Wei and Guoxiang Gu
}

\begin{abstract}
In the existing works on distributed detection in sensor networks, local sensor nodes either quantize the observation or directly scale the analog observation and then transmit the processed information independently over wireless channels to a fusion center. In this paper, we exploit the advantages of both of these two approaches by constructing an equivalent $\Sigma-\Delta$ ADC across space over wireless sensor networks. Sensors are arranged in a mixing of parallel and serial topologies, enabling each sensor to transmit binary information to the fusion center, while in the meantime preserving the analog information through collaborative processing. Comparison with existing approaches demonstrates the superiority of our proposed scheme in both AWGN and fading channels in terms of the resulting detection error probability.
\end{abstract}

\section{INTRODUCTION}

Distributed detection with multiple sensors has received great attentions over decades ( See [1], [2], [5] and references therein). In a distributed sensor network, multiple sensors work collaboratively to distinguish multiple hypothesis. Each local sensor performs some preliminary data processing and may send the information to other sensors. Ultimately the locally processed information is collected by a fusion center where an optimal or a suboptimal fusion algorithm is performed to reach a final decision.

Unlike the traditional distributed detection works where sensor output is assumed immediately available to the fusion center, most of the recent works on distributed detection explicitly take the wireless channels between sensors and the fusion center into consideration [5], [3], [4]. Parallel topology is usually adopted in these works, where sensors transmit information to the fusion center via independent orthogonal channels without exchanging any information between them. The objective is to design optimal local processing rules with ([5]) or without using channel information, as well as associated fusion algorithms under certain performance criterion (e.g. Bayesian or Neyman-Pearson criterion). Analog transmission (scale and forward scheme) has also been investigated for additive white Gaussian noise(AWGN) channels [4].

In this paper, we propose a novel distributed detection scheme for binary hypothesis test. Inspired by the concept of analog to digital converter (ADC) which converts analog signals to bit-stream, we propose a novel $\Sigma-\Delta$ ADC-based distributed detection scheme where wireless sensor nodes

\footnotetext{
${ }^{1}$ This paper was supported in part by the Board of Regents of Louisiana under grants LEQSF(2004-07)-RD-A-17

${ }^{2}$ The authors are with the Department of Electrical and Computer Engineering, Louisiana State University, Baton Rouge, LA 70803. E-mail: dwang3@1su.edu, \{swei, ggu\} @ece.lsu.edu.
}

jointly process the analog observations using $\Sigma-\Delta$ modulation without oversampling and decimation. Due to the limited power budgets, each local sensor is only allowed to take one sample of observation and send one bit message to the fusion center. Furthermore, each sensor is allowed to communicate to its next adjacent sensor. This novel combination of serial and parallel topology [1] enables us to form an equivalent $\Sigma-\Delta$ loop across space within the wireless sensor network. As shown in our simulation results, the ADC-based distributed detection system outperforms both the binary and analog approaches in both AWGN channels and fading channels under certain conditions and it can be used to achieve better performance in certain applications that a large number of sensors are deployed closely together, so that the additional power consumed for inter-sensor communication is relatively small.

\section{SYSTEM MODEL AND PROBLEM FORMULATION}

\section{A. System Model}

In this paper, we focus on a distributed binary hypothesis testing problem. Suppose that there are $N$ sensor nodes observing a random phenomenon. Each sensor collects only one noisy observation described by

$$
\begin{array}{lll}
H_{1}: & x_{i}=s+w_{i}, & i=1,2, \cdots, N \\
H_{0}: & x_{i}=w_{i}, & i=1,2, \cdots, N
\end{array}
$$

where $s$ is a known constant signal and $\left\{w_{1}, w_{2}, \cdots, w_{N}\right\}$ are measurement noises that are mutually independent and identically distributed as real Gaussian random variables with mean zero and variance $\sigma_{w}^{2}$. Different from distributed detection systems with parallel topology [1], we also allow communication between adjacent sensor nodes. As a result, the $N$-sensor actually follows a mixing of serial and parallel topology as shown in Figure 2. For this topology, sensor node $i$ maps its local observation $x_{i}$ and the signal $v_{i-1}$ sent by the adjacent sensor node $i-1$ to its output $y_{i}=\gamma_{i}\left(x_{i}, v_{i-1}\right)$, which is then transmitted to a fusion center over a unique assigned channel (e.g. a time slot) that experiences independent flat fading with respect to other orthogonal channels. The received signal at the fusion center from the $i$ th sensor node is given by

$$
\hat{y}_{i}=h_{i} y_{i}+n_{i}
$$

where $n_{i}$ is AWGN with variance $\sigma_{n}^{2}$ and $h_{i}$ is the fading channel gain with Rayleigh distribution and $E\left(\left|h_{i}\right|^{2}\right)=1$. We also assume non-coherent detection at fusion center where 


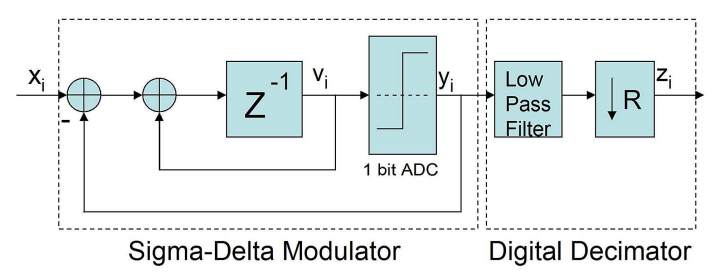

Fig. 1. First order $\Sigma-\Delta$ modulator ADC system

only channel fading statistics instead of global channel state information (CSI) are available.

The fusion center receives $\left\{\hat{y}_{1}, \hat{y}_{2}, \cdots, \hat{y}_{N}\right\}$ and makes a global decision $\theta$ based on an optimal or suboptimal fusion rule which will be discussed in the next two sections. Denote the prior probability by $\pi_{i}=P\left(H_{i}\right), i=0,1$. The detection performance is characterized by the detection error probability

$$
P_{e, N}=\pi_{0} P\left(\theta=H_{1} \mid H_{0}\right)+\pi_{1} P\left(\theta=H_{0} \mid H_{1}\right)
$$

with respect to the sensor measurement SNR denoted as $P_{s} \triangleq s^{2} / \sigma_{w}^{2}$ and the inter-sensor-center channel SNR (with normalized transmission power $1 / \sigma_{n}^{2}$ ) denoted by $P_{t}=1 / \sigma_{n}^{2}$. The goal is to minimize the detection error probability by designing the proper local transmission strategy $\left\{\gamma_{i}\right\}_{i=1}^{N}$ and the corresponding fusion rule $\gamma_{0}$ at the fusion center. With the statistic knowledge of $w_{i}, h_{i}, n_{i}$, the optimal fusion rule at the fusion center is the maximum a posteriori probability (MAP) decision

$$
\gamma_{0}\left(\hat{y}_{1}, \cdots, \hat{y}_{N}\right)=\left\{\begin{array}{l}
H_{0}, \Lambda \geq \frac{\pi_{1}}{\pi_{0}}, \\
H_{1}, \Lambda<\frac{\pi_{1}}{\pi_{0}} .
\end{array}\right.
$$

where $\Lambda=\frac{f\left(\hat{y}_{1}, \cdots, \hat{y}_{N} \mid H_{0}\right)}{f\left(\hat{y}_{1}, \cdots, \hat{y}_{N} \mid H_{1}\right)}$ is the likelihood ratio (LR) of the joint probability density function (pdf) of $\left\{\hat{y}_{i}\right\}_{i=1}^{N}$ under each hypothesis.

\section{B. $\Sigma-\Delta$ modulation $A D C$}

A block diagram of a conventional first order $\Sigma-\Delta$ ADC system is shown in Fig. 1 which includes a $\Sigma-\Delta$ modulator, followed by a digital decimator. The modulator consists of an integrator, an internal $\mathrm{AD}$ converter or quantizer, and a feedback path. The relationship between the input and output of the $\Sigma-\Delta$ modulator is given by

$$
v_{i+1}=x_{i}-y_{i}+v_{i}, \quad y_{i+1}=q\left(v_{i+1}\right) .
$$

where

$$
q(v)=\left\{\begin{aligned}
1, & v \geq 0 \\
-1, & v<0
\end{aligned}\right.
$$

assuming that the output of the one bit quantizer is either 1 or -1 . Using a linear model [6], the $\mathrm{Z}$ domain relationship of the $\Sigma-\Delta$ modulator is:

$$
Y(z)=X(z) z^{-1}+E(z)\left(1-z^{-1}\right)
$$

where $Y, X$ and $E$ are the $\mathrm{Z}$ transforms of the output, input signal and the quantization error process, respectively. The one bit quantization error is shaped by a first order differentiator or

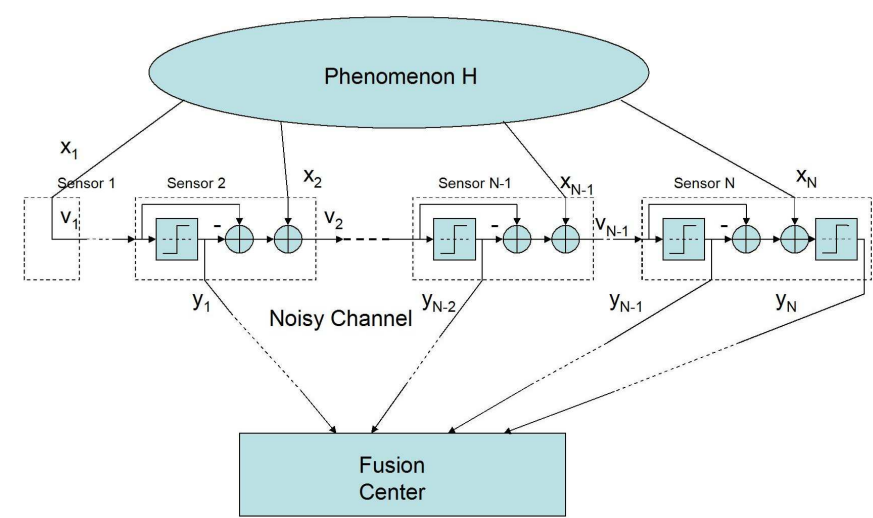

Fig. 2. $\Sigma-\Delta$ ADC based distributed detection scheme

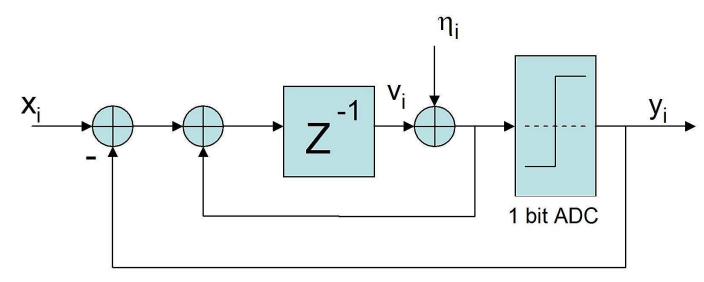

Fig. 3. Equivalent model for $\Sigma-\Delta \mathrm{ADC}$ based distributed detection system

high-pass filter. For conventional $\Sigma-\Delta \mathrm{ADC}$, the continuoustime signal is first oversampled before being fed into the $\Sigma-\Delta$ modulator. The oversampling process spreads the fixed quantization noise power over a bandwidth that is much larger than the signal band. The noise spectrum is further shaped by the noise transfer function in order to push most of the quantization noise power outside the signal bandwidth [6]. Consequently, the quantization noise can be significantly reduced by the low-pass filter with cut off frequency equal to the signal bandwidth. The digitalized signal is effectively downsampled to the signal Nyquist rate. The output of the digital decimator thus becomes a multi-bit finite-valued digital data reconstructing the analog input.

\section{C. $\Sigma-\triangle A D C$ based distributed detection system}

We now consider integrating the $\Sigma-\Delta$ modulator into the sensor node as a local quantizer with only 1 bit resolution. The fusion center uses the output bit stream of the modulator that represents the analog observation to make a binary decision. For the detection purpose, we drop the oversampling block in the $\Sigma-\Delta$ modulator due to the following reason. Our purpose is not to reconstruct the analog input signal $x_{i}$, but to distinguish between two hypothesis. Without oversampling, the spectrum of the measurement noise in $x_{i}$ is spread in the same way as the quantization noise spectrum, most of which will be filtered by the subsequent decimator.

Fig. 2 shows the $\Sigma-\Delta$ ADC based distributed detection scheme and Fig. 3 shows its equivalent model. Assume each sensor node takes only one sample of $x_{i}$. Since the time domain approach of the $\Sigma-\Delta$ modulation can be transformed to space domain due to the invariance of the signal, we 
implement the $\Sigma-\Delta$ modulation in the distributed sensor network with a combination of serial and parallel topology [1]. The $i$ th sensor node transmits its $v_{i}$ to the $i+1$ th sensor node over an inter-sensor AWGN channel with power $P_{I}$ and

$$
P_{I}=\frac{E\left(v_{i}^{2}\right)}{\sigma_{\eta}^{2}}
$$

where $E\left(v_{i}^{2}\right)$ will be given in Section 3 and $\sigma_{\eta}^{2}$ is the variance of AWGN $\eta_{i}$. We assume no fading in the intersensor channels because of the close distance between adjacent sensor nodes. At the $i+1$ th sensor node, $v_{i}+\eta_{i}$ is quantized to obtain $y_{i}$. The $i+1$ th sensor uses $v_{i}+\eta_{i}$ and $y_{i}$ as the feedback to generate $v_{i+1}$, meanwhile transmitting $y_{i}$ to the fusion center and $v_{i+1}$ to the next sensor node. Therefore we form an equivalent $\Sigma-\Delta$ modulator loop within the sensor network. The fusion center will use $\left\{\hat{y}_{1}, \hat{y}_{2}, \cdots, \hat{y}_{N}\right\}$ to perform detection.

\section{Distributed Detection IN AWGN CHANNELS}

We first investigate our proposed scheme in AWGN channels and develop a sub-optimal detection algorithm. A closedform solution to the detection error probability is obtained for this algorithm, which is compared with that for binary and analog approaches under the parallel topology.

WLOG, we assume $\pi_{0}=\pi_{1}$. The optimal MAP fusion rule requires the joint pdf of $y_{i}$ and $\hat{y}_{i}$ in order to compute the likelihood ratio in Eq.(3). The computation of the joint density function in Eq.(3) involves multiple integration and does not yield any insight regarding the performance discrepancy between different systems. We therefore adopt a suboptimal fusion rule with close to optimal detection performance [9]. In [8], it was shown that averaging $\left\{y_{i}\right\}_{i=1}^{N}$ is an efficient decoder that functions as a digital decimator for single loop $\Sigma-\Delta$ modulators with i.i.d Gaussian input. This result inspires us to employ $Z:=\frac{1}{N} \sum_{i=1}^{N} \hat{y}_{i}$ as our detection statistics. The averaging of $\left\{\hat{y}_{i}\right\}_{i=1}^{N}$ is compared with a threshold which will be shown to be $s / 2$ later. Although the results in [8] are obtained for traditional $\Sigma-\Delta$ ADC with i.i.d Gaussian inputs, our simulation results demonstrate it is a good approximation when inter-sensor SNR is reasonably high or even when the measurement noise are correlated [9].

For AWGN channel with $h_{i}=1$ in (1), we thus have

$$
Z=\frac{1}{N} \sum_{i=1}^{N}\left(y_{i}+n_{i}\right)
$$

where $n_{i}$ is the noise of channel from sensor node to the fusion center with variance $\sigma_{n}^{2}=1 / P_{t}$.

Applying the assumed input signal model, we rewrite (4) and obtain

$$
v_{i+1}=v_{i}-q\left(v_{i}\right)+m+w_{i}
$$

where $m=0$ under $H_{0}$ and $m=s$ under $H_{1}$ and the desired $\operatorname{LR} \frac{f\left(Z \mid H_{0}\right)}{f\left(Z \mid H_{1}\right)}=\frac{f(Z \mid m=0)}{f(Z \mid m=s)}$. Denote $\lambda(v):=v-q(v)+m$. We can transform the quantization error by defining $\bar{e}_{i}:=\lambda\left(v_{i}\right)$. Now (8) can be written as $v_{i}=\bar{e}_{i-1}+w_{i-1}$ that yields the recursion: $\bar{e}_{i+1}=\lambda\left(\bar{e}_{i}+w_{i}\right)$. It is also proved in [8] that the process $\overline{\mathbf{e}}=\left\{\bar{e}_{n-1} \mid n=0,1, \ldots\right\}$ is a real valued discretetime Markov process and it has a unique invariant probability measure if and only if $|m|<1$. Note for $|m| \geq 1$, we can alway scale accordingly the quantization level in (5) to make the equivalent $m<1$. WLOG, we assume $|m|<1$ in the rest of this paper.

Moreover, $\bar{e}_{i}$ can be split into two independent random variables

$$
\bar{e}_{i}=g_{i}+m+o_{i}
$$

The random variable $g_{i}$ is referred as"granular noise" which is uniformly distributed over the interval $[-1,+1]$, and $o_{i}$ as "slope overload noise". In the frequency domain, $o_{i}$ is concentrated in the high-frequency end of the spectrum while $g_{i}$ is concentrated in the low-frequency end, which is referred as granular mode and slope overload mode respectively. We now assume that the $\Sigma-\Delta$ modulation in distributed detection is under granular mode when the subsequent decimator can eliminate most of the quantization noise power.

For the distributed detection scheme in Fig. 2 and Fig. 3, after including inter-sensor channels, we can modify (8) as

$$
v_{i+1}=v_{i}+\eta_{i}-q\left(v_{i}+\eta_{i}\right)+m+w_{i}
$$

where $\eta_{i}$ is the white Gaussian noise in the inter-sensor channel with variance $\sigma_{\eta}^{2}=E\left(v_{i}^{2}\right) / P_{t}$ and $E\left(v_{i}^{2}\right)=E\left(\bar{e}_{i}^{2}\right)+\sigma_{w}^{2}=$ $1 / 3+s^{2} / 2+\sigma_{w}^{2}$. The binary quantizer error $\bar{e}_{i}$ is modified as

$$
\bar{e}_{i}=v_{i}+\eta_{i}-q\left(v_{i}+\eta_{i}\right)+m .
$$

Since we are interested in the decimator output $Z$, combining (7),(9) and (10) yields

$$
\bar{n}=\frac{1}{N}\left[\sum_{i=0}^{N-1}\left(w_{i}+\eta_{i}+n_{i}\right)\right]+\frac{1}{N}\left(\bar{e}_{0}-\bar{e}_{N}\right),
$$

where $\bar{n}:=Z-m$ is the detection statistics. The first term in (11) is a zero-mean Gaussian random variable with variance $\sigma^{2}=\frac{1}{N}\left(\sigma_{w}^{2}+\sigma_{\eta}^{2}+\sigma_{n}^{2}\right)$ where $\sigma_{w}^{2}+\sigma_{\eta}^{2}+\sigma_{n}^{2}$ is referred as the total noise power. Under granular mode $\bar{e}_{0}-\bar{e}_{N}$ can be replaced by $g_{0}-g_{N}$. When $N$ is large enough such that the correlation between $g_{0}$ and $g_{N}$ is very weak, $E:=\frac{1}{N}\left(\bar{e}_{0}-\bar{e}_{N}\right)$ becomes the sum of two i.i.d random variables uniformly distributed over $[-1 / N, 1 / N]$ and its pdf is a triangular waveform given by [9]

$$
f_{E}(x)=\left\{\begin{aligned}
\frac{1}{4} N^{2} x+\frac{N}{2}, & x \in[-2 / N, 0], \\
-\frac{1}{4} N^{2} x+\frac{N}{2}, & x \in[0,2 / N] .
\end{aligned}\right.
$$

Since the noise $w_{i}, \eta_{i}, n_{i}$ are mutually independent and are all independent of $\bar{e}_{i}$. The pdf of $\bar{n}$ is thus the convolution of a zero-mean Gaussian pdf with variance $\sigma^{2}$, denoted as $\Psi(x)$ and the triangular waveform function $f_{E}(x)$,

$$
\begin{aligned}
f_{\bar{n}}(y)= & \int_{-\infty}^{\infty} \Psi(x) f_{E}(y-x) d x \\
= & \frac{N^{2} \sigma}{4 \sqrt{2 \pi}}\left[e^{\frac{-\left(y-\frac{2}{N}\right)^{2}}{2 \sigma^{2}}}+e^{\frac{-\left(y+\frac{2}{N}\right)^{2}}{2 \sigma^{2}}}-2 e^{\frac{-y^{2}}{2 \sigma^{2}}}\right] \\
& +\frac{N^{2}}{4}\left[2 y Q\left(\frac{y}{\sigma}\right)-\left(y-\frac{2}{N}\right) Q\left(\frac{(y-2 / N)}{\sigma}\right)\right] \\
& -\frac{N^{2}}{4}\left(y+\frac{2}{N}\right) Q\left(\frac{(y+2 / N)}{\sigma}\right)
\end{aligned}
$$

where $Q(x)=\frac{1}{\sqrt{2 \pi}} \int_{x}^{\infty} e^{-\frac{t^{2}}{2}} d t$. 
It can be seen that $f_{\bar{n}}(y)$ is an even function. Consequently, the pdf of $Z$ under each hypothesis is $f_{\bar{n}}(Z-m)$, which is concentrated over 0 or $s$. The standard LR test (LRT) rule gives us the detection threshold $y_{0}=s / 2$ and the detection error probability $P_{e}$ is

$$
P_{e}=\int_{y_{0}}^{\infty} f_{\bar{n}}(y) d y=\int_{y_{0}}^{\infty} \int_{-\infty}^{\infty} \Psi(x) f_{E}(y-x) d x d y .
$$

The result of the integral yields [9]

$$
P_{e}=\frac{N^{2}}{8} A+\frac{N^{2} \sigma}{8 \sqrt{2 \pi}} B
$$

where $A=\left[\left(y_{0}-\frac{2}{N}\right)^{2}+\sigma^{2}\right] Q\left(\frac{\left(y_{0}-2 / N\right)}{\sigma}\right)+\left[\left(y_{0}+\frac{2}{N}\right)^{2}+\right.$ $\left.\sigma^{2}\right] Q\left(\frac{\left(y_{0}+2 / N\right)}{\sigma}\right)-2\left(y_{0}^{2}+\sigma^{2}\right) Q\left(\frac{y_{0}}{\sigma}\right)$ and $B=2 y_{0} e^{\frac{-y_{0}^{2}}{2 \sigma^{2}}}-$ $\left(y_{0}-\frac{2}{N}\right) e^{\frac{-\left(y_{0}-\frac{2}{N}\right)^{2}}{2 \sigma^{2}}}-\left(y_{0}+\frac{2}{N}\right) e^{\frac{-\left(y_{0}+\frac{2}{N}\right)^{2}}{2 \sigma^{2}}}$.

We next compare the detection performance of our proposed scheme under the mixing topology with that of binary and analog approaches under the parallel topology for sensor nodes. In binary distributed detection systems under parallel topology, each sensor node locally makes a binary decision by comparing the measurement $x_{i}$ with the threshold of $s / 2$ and then transmits the binary message to the fusion center. The LR of $\left\{\hat{y}_{i}\right\}_{i=1}^{N}$ at the fusion center under two hypothesis can be easily derived as,

$$
\begin{aligned}
\Lambda & =\prod_{i=1}^{N} \frac{f\left(y_{i} \mid H_{0}\right)}{f\left(y_{i} \mid H_{1}\right)} \\
& =\prod_{i=1}^{N} \frac{P_{f} e^{-\frac{\left(\hat{y}_{i}-1\right)^{2}}{2 \sigma_{n}^{2}}}+\left(1-P_{f}\right) e^{-\frac{\left(\hat{y}_{i}+1\right)^{2}}{2 \sigma_{n}^{2}}}}{P_{f} e^{-\frac{\left(\hat{y}_{i}+1\right)}{2 \sigma_{n}^{2}}}+\left(1-P_{f}\right) e^{-\frac{\left(\hat{y}_{i}-1\right)}{2 \sigma_{n}^{2}}}}
\end{aligned}
$$

where $P_{f}=Q\left(s / 2 \sigma_{w}\right)$ is the local detection error probability. The optimal LRT fusion rule is thus straightforward to implement. Simulation results for binary system detection performance will be provided in Section 5.

The second type of transmission mapping strategy at local sensor is that each sensor node retransmits a scaled version of its own analog observation. Detection error probability can be written explicitly as [9],

$$
P_{e, A}=Q\left(\frac{\alpha s / 2}{\sqrt{\left(\alpha^{2} \sigma_{w}^{2}+\sigma_{n}^{2}\right) / N}}\right)
$$

where $\alpha=\sqrt{\frac{2}{s^{2}+2 \sigma_{w}^{2}}}$ is the scaling factor. Comparison of the three schemes will be discussed in Section 5 .

\section{Distributed Detection IN FADING CHANNELS}

For non-coherent detection in fading channels, it is generally impossible to obtain a closed-form formula for the detection error probability with finite number of sensors. We will provide an outline of the LRT-based optimal fusion rule for the three approaches and evaluate their performances by simulations.

Binary case:
Assume $h_{i}$ is Rayleigh distributed with $E\left(h_{i}^{2}\right)=1$. For binary transmission, the desired LR is

$$
\Lambda=\prod_{i=1}^{N} \frac{P_{f} f\left(\hat{y}_{i} \mid y_{i}=1\right)+\left(1-P_{f}\right) f\left(\hat{y}_{i} \mid y_{i}=-1\right)}{P_{f} f\left(\hat{y}_{i} \mid y_{i}=-1\right)+\left(1-P_{f}\right) f\left(\hat{y}_{i} \mid y_{i}=1\right)}
$$

where $f\left(\hat{y}_{i} \mid y_{i}=1\right)=\int_{0}^{\infty} 2 x e^{-x^{2}} \frac{1}{\sqrt{2 \pi} \sigma_{n}} e^{-\frac{(x-y)^{2}}{2 \sigma_{n}{ }^{2}}} d x$

Analog case:

For analog system, $y_{i}=\alpha x_{i}$, pdf of $\hat{y}_{i}$ given $\alpha x$ is

$$
f\left(\hat{y}_{i} \mid y_{i}=\alpha x\right)=\int_{0}^{\infty} 2 \frac{z}{\alpha x} e^{-\left(\frac{z}{\alpha x}\right)^{2}} \frac{1}{\sqrt{2 \pi} \sigma_{n}} e^{-\frac{(z-y)^{2}}{2 \sigma_{n}^{2}}} d z
$$

The pdf of $\hat{y}_{i}$ given $H_{0}$ is

$$
f\left(\hat{y}_{i} \mid H_{0}\right)=\int_{-\infty}^{\infty} \frac{1}{\sqrt{2 \pi} \sigma_{w}} e^{-\frac{(x-s)^{2}}{2 w^{2}}} f\left(\hat{y}_{i} \mid y_{i}=\alpha x\right) d x
$$

The rest of the procedure to obtain the LR is exactly the same as the binary case.

$\Sigma-\Delta$ case:

For simplicity, we only consider the suboptimal fusion rule here where the received signals are demodulated first instead of using the soft information. It is shown in [7] that the joint probability of $\left\{y_{i}\right\}_{y=1}^{N}$ can be obtained from (4) by an iterative computation of

$$
p_{y_{i}}=\int_{0}^{\infty} f_{v_{i}}(v) d v
$$

and

$$
f_{v_{i+1}}(v)=f_{x_{i}}(v) * f_{e_{i}}(-v)
$$

where $e_{i}=q\left(v_{i}\right)-v_{i} \cdot f_{e_{i}}(-v)$ is obtained by,

$$
f_{e_{i}}(-e)=u(e+1) f_{v_{i}}(e+1)+u(-e+1) f_{v_{i}}(e-1)
$$

in which $u(e)$ is the step function defined as 0 for $e<0$ and 1 for $e \geq 0$. We apply this approach as an approximation since it does not consider inter-sensor channels. The communication bit error probability over Rayleigh fading channel $p\left(\hat{y}_{i} \neq y_{i}\right)=\frac{1}{2}\left(1-\sqrt{\frac{P_{t}}{1-P_{t}}}\right)$. The LR at the fusion center can be derived as,

$$
\Lambda=\frac{\sum_{y_{1}, \cdots, y_{N}} \prod_{i=1}^{N} p\left(\hat{y}_{i} \mid y_{i}\right) p\left(y_{1}, \cdots, y_{N} \mid H_{0}\right)}{\sum_{y_{1}, \cdots, y_{N}} \prod_{i=1}^{N} p\left(\hat{y}_{i} \mid y_{i}\right) p\left(y_{1}, \cdots, y_{N} \mid H_{1}\right)}
$$

where $p\left(y_{1}, \cdots, y_{N} \mid H_{i}\right)$ can be calculated from the joint pdf $f\left(v_{1}, \cdots, v_{N} \mid H_{i}\right)$, which can be found by working out the iterative process in (16). The optimal detector using hard decision information on $\left\{\hat{y}_{i}\right\}_{i=1}^{N}$ can thus be derived straightforwardly like the previous two cases.

\section{NUMERICAL AND SIMULATION RESULTS}

In this section we compare the performance of $\Sigma-\Delta$ ADC based distributed detection system with that of binary and analog system of same parameters $\left(N, P_{s}, P_{t}\right)$ in terms of the detection error probability.

Fig. 5 illustrates the detection performance of three distributed detection systems as a function of $P_{s}$ in AWGN channels. From this figure we can see that $\Sigma-\Delta$ ADC based distributed detection system can outperform the analog distributed detection system. To better understand this, let us 


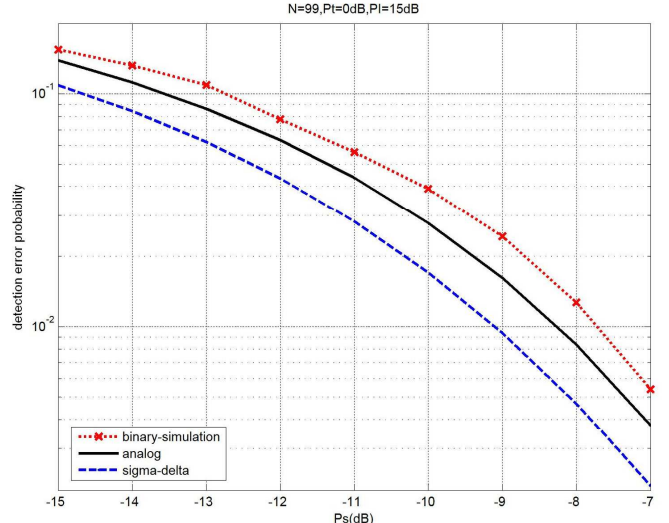

Fig. 4. Detection error probability versus $P_{s}$ in AWGN channels with $\mathrm{N}=99, P_{t}=0 \mathrm{~dB}, P_{I}=15 \mathrm{~dB}$, s=0.6

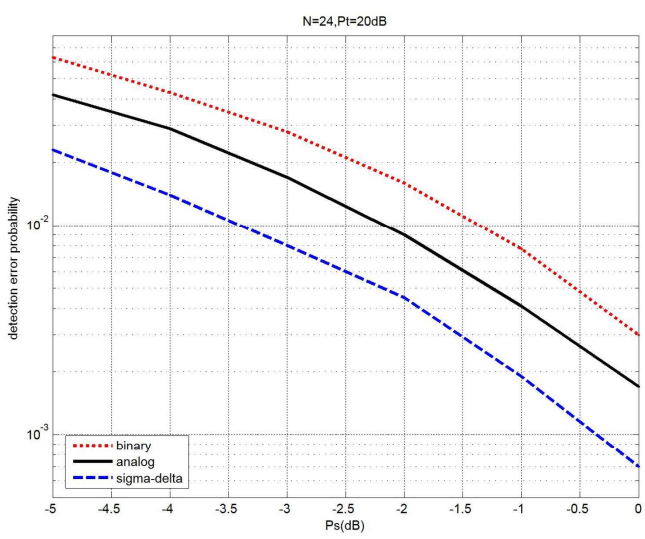

Fig. 5. Simulation results for detection error probability versus $P_{s}$ in Rayleigh fading channels with $\mathrm{N}=24, P_{t}=20 \mathrm{~dB}, P_{I}=15 \mathrm{~dB}$

neglect $\bar{e}_{0}-\bar{e}_{N}$ and inter-sensor channel noise $\eta_{i}$ in (11). This is reasonable when $\sigma_{n}^{2}, \sigma_{w}^{2}$ and $N$ are large, while sensor nodes are close to each other. Hence the detection symbol $Z$ could be approximated as $m+\frac{1}{N}\left[\sum_{i=0}^{N-1}\left(w_{i}+n_{i}\right)\right]$. This is exactly the detection statistics for analog system when scale factor $\alpha=1$. Since detection error probability in (14) increases as $\alpha$ decreases, it is easy to see that $\Sigma-\Delta$ system will outperform the analog system when $\alpha<1$, i.e. $s^{2} / 2+\sigma_{w}^{2}>1$. It can also be intuitively explained from the fact that binary sensor nodes would cause significant distortion during the 1-bit local compression when $P_{s}$ is low yet more robust than analog system when $P_{t}$ is low [3]. $\Sigma-\Delta$ system reserve the raw observations as analog sensors but transmit the message as binary sensors, therefore combine the advantages of the two traditional approaches.

Fig. 6 and Fig. 7 show the simulation results for detection performance as a function of $P_{s}$ and $P_{t}$ respectively in Rayleigh fading channels. $\Sigma-\Delta$ ADC based distributed detection appears to be more robust against fading because of the correlations between $\left\{y_{i}\right\}_{i=1}^{N}$, especially in high channel SNR case. Performance degradation in low channel SNR region (in Fig. 7) is due to the hard decision we use in fusion decision since communication bit error probability increases rapidly as channel SNR falls. In [9], we have investigated the LRT fusion rule based on soft decision for the $\Sigma-\Delta$

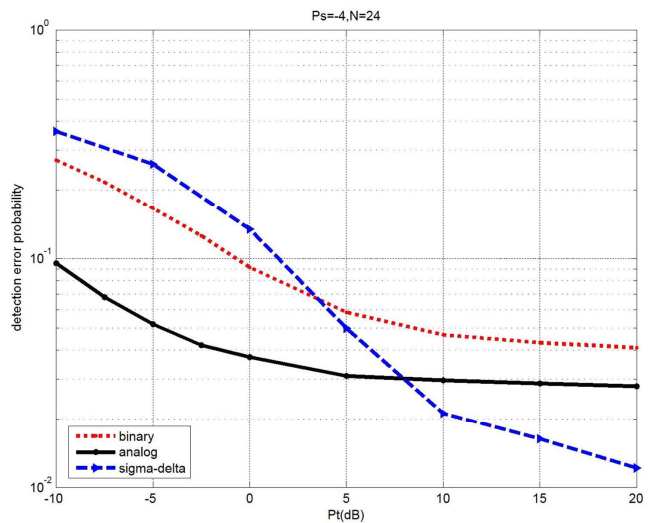

Fig. 6. Simulation results for detection error probability versus $P_{t}$ in Rayleigh fading channels with $\mathrm{N}=24, P_{s}=-4 \mathrm{~dB}, P_{I}=15 \mathrm{~dB}$

modulation based distributed detection system. We need to point out that although input signal statistics is assumed i.i.d in this paper for simplicity purpose, the proposed approach can be easily extended to non-i.i.d sources [9].

In addition, our proposed scheme improves the detection performance at the cost of the extra communication links between adjacent sensors which will increase the complexity, power consumption and time delay of the system compared to those of the existing parallel distributed detection schemes. The question is that given the total power budget, how much should we spend on the inter-sensor communication to keep the overall performance still better than the analog and binary systems with the same power budget spent solely on the communication between sensors and fusion center? We will investigate this in our future work. Nevertheless, our preliminary study gives us motivating examples of the trade off between performance and complexity in distributed detections, as the $\Sigma-\Delta$ system is a special example of such systems in which sensors collaboratively process the observations.

\section{REFERENCES}

[1] R. Viswanathan and P. K. Varshney, "Distributed detection with multiple sensors: part I: fundamentals," Proc.IEEE, vol. 85, no. 1, pp. 54, Jan. 1997.

[2] R. S. Blum, S. A. Kassam, And H. V. Poor, "Distributed detection with multiple sensors: part II: advanced topics," Proc.IEEE, vol. 85, pp. 64, Jan. 1997.

[3] R. Niu, B. Chen, and P. K. Varshney, "Fusion of decisions transmitted over Rayleigh fading channels in wireless sensor networks," IEEE Trans. Signal Process., vol. 54, no. 3, pp.1018, Mar. 2006.

[4] J. Chamberland and V. Veeravalli, "Asymptotic results for decentralized detection in power constrained wireless sensor networks," IEEE J. Select. Areas Commun., vol. 22, pp. 1007, Aug. 2004.

[5] B. Chen, L. Tong, and P. K. Varshney, "Channel-aware distributed detection in wireless sensor networks," IEEE Signal Processing Mag., pp. 16, Jul. 2006.

[6] P. M. Aziz, H. V. Sorensen and J. V. Spiegel, "An overview of Sigma-Delta converters: How a 1-Bit ADC achieves more than 16-Bit Resolution," IEEE Signal Processing Mag., pp. 61, Jan. 1996.

[7] S. Hoyos, B.M. Sadler, G.R. Arce, "Monobit digital receivers for ultrawideband communications," IEEE Trans. Wireless Commun., vol. 4, pp. 1337, Jul. 2005.

[8] T. Koski, "Statistics of the binary quantizer error in single-Loop SigmaDelta modulation with white Gaussian input," IEEE Trans. Signal Process., vol. 41, no. 4, pp. 931, Jul. 1995.

[9] D. Wang, "Sigma-Delta Modulation Based Distributed Detection in Wireless Sensor Networks", M.S. Thesis at LSU, July 2007. 